\title{
An Interactive Graphical Environment for Code Optimization
}

\author{
Jie Tao ${ }^{1}$, Thomas Dressler ${ }^{2}$, and Wolfgang Karl $^{2}$ \\ ${ }^{1}$ Institut für Wissenschaftliches Rechnen \\ Forschungszentrum Karlsruhe \\ 76021 Karlsruhe, Germany \\ jie.tao@iwr.fzk.de \\ ${ }^{2}$ Institut für Technische Informatik \\ Institut für Technische InformatiK \\ 76128 Karlsruhe, Germany \\ karl@ira.uka.de
}

\begin{abstract}
Applications usually do not show a satisfied initial performance and require optimization. This kind of optimization often covers a complete process, starting with gathering performance data, followed by performance visualization and analysis, up to bottleneck finding and code modification. In this paper we introduce DECO (Development Environment for Code Optimization), an interactive graphical interface that enables the user to conduct this whole process within a single environment.
\end{abstract}

Keywords: Performance tools, visualization, cache optimization.

\section{Introduction}

General-purpose architectures are not tailored to applications. As a consequence, most applications usually do not show high performance as initially running on certain machines. Therefore, applications often have to be optimized for achieving expected performance metrics.

This kind of optimization, however, is a quite tedious task for users and as a consequence various tools have been developed for providing support. First, users need a performance analyzer [1] or a visualization tool [7] capable of presenting the execution behavior and performance bottlenecks. These tools have to rely on profilers [4], counter interfaces [3], or simulators [6] to collect performance data. Hence, tools for data acquisition are required. In addition, users need platforms to perform transformations on the code. These platforms can be integrated in the analysis tool but usually exist as an individual toolkit.

In summary, users need the support of a set of different tools. Actually, most tool vendors provide a complete toolset to help the user step-by-step conduct the optimization process, from understanding the runtime behavior to analyzing performance hotspots and detecting optimization objects. Intel, for example, has developed VTune Performance Analyzer for displaying critical code regions and 
Thread Profiler for presenting thread interaction and contention [5]. The Center for Information Services and High Performance Computing at the University of Drednen developed Vampir 20 for performance visualization and Goofi [8] for supporting loop transformation in Fortran programs.

Similarly, we implemented a series of tools for cache locality optimization. This includes a cache visualizer [9] which demonstrates cache problems and the reason for them, a data profiler which collects information about global cache events, a cache simulator [10] for delivering runtime cache activities, and a pattern analysis tool [1] for discovering the affinity among memory operations.

By applying these tools to optimize applications, we found that it is inconvenient to use them separately. In this case, we developed the program development environment DECO in order to give the user a single interface for conducting the whole process of code tuning. A more challenging motivation for us to develop DECO is the user requirement of a platform to work with their programs. This platform must provide all necessary functionality for building and running an application. The third reason for a program development environment is that users need to compare the execution time or other performance metrics of different runs with both unoptimized and optimized code versions. It is more flexible if users could acquire this information within a single view than having to switch across several windows.

These features are general and can be applied directly or with slight extension by other tool developers to build a development environment for their own need. For our purpose with cache locality optimization DECO has an additional property: it visualizes the output of the pattern analysis tool and maps the access pattern to the source code.

The rest of the paper is organized as follows. We first describe the design and implementation of DECO in Section 2. In Section 3 we present its specific feature for cache locality optimization, together with some initial optimization results with small codes. In Section 4 the paper is concluded with a short summary and some future directions.

\section{DECO Design and Implementation}

The goal of DECO is to provide program developers an easy-to-use environment for step-by-step running the program, analyzing the performance, conducting optimization, executing the program again, and then studying the impact of optimizations. This is actually a feedback loop for continuous optimization. Users repeat this loop till a satisfied performance is achieved. For giving the user a simple view DECO provides all needed functionality for the whole loop in a single window.

As depicted in Figure 1 this window consists of a menu bar and three visualization areas. The menu bar is located on the top of the window, with items for file operation, view configuration, project creation, executable building, and performance analyzing. The left field under the menu bar is a code editor, where the program source is displayed in different color for a better overview and an 


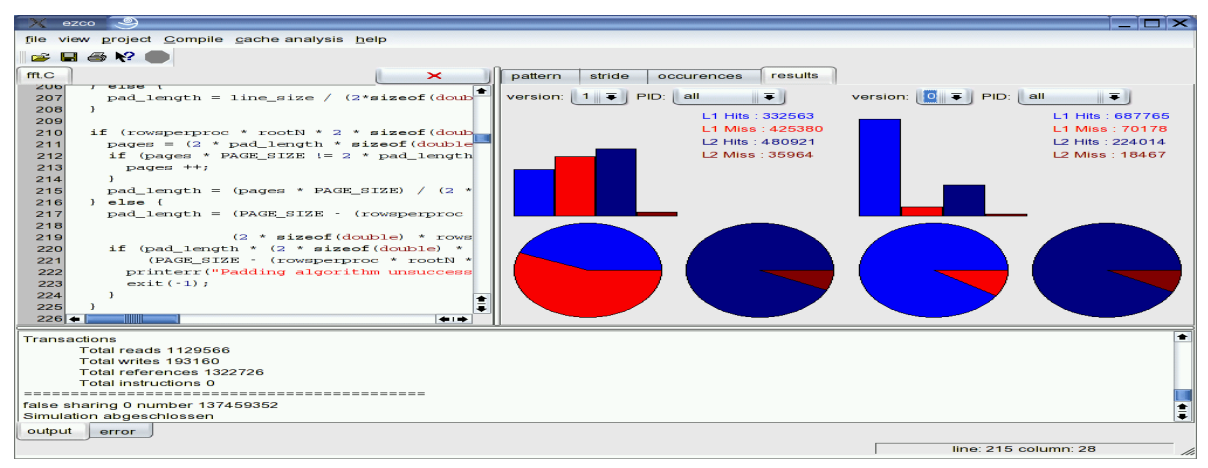

Fig. 1. Main window of the program development environment

easy logic analysis. Next to the editor is a subwindow for demonstrating runtime results, such as execution time, cache miss statistics, communication overhead (e.g. for MPI programs), and analysis of specific constructs (e.g. parallel and critical regions in OpenMP programs). In addition, the visualization of access pattern, for our special need of cache locality optimization, is combined with this subwindow. The output and error reports delivered during the run of applications or tools are displayed at the bottom.

Building Projects. DECO targets on realistic applications which are usually comprised of several files including head and Makefile. Hence, applications are regarded as project within DECO. Projects must be created for the first time and then can be loaded to the DECO environment using corresponding options in the menu bar.

Figure 2 shows two sample subwindows for creating a project. First users have to give the project a name and the path where the associated files are stored. Then users can add the files that need analysis and potentially optimization into the project by simply select the object from the files at the given path, as illustrated on the top picture of figure 2. It is allowed to concurrently open a set of files, but only one file is active for editing.

After modification of the source program users can generate an executable of the application using Menu Compile that contains several options like make and make clean, etc. Commands for running the application are also included.

Tool Integration. DECO allows the user to add supporting tools into the environment. To insert a tool, information about the name, the path, and configuration parameters has to be provided. For this, DECO lists the potential parameters that could be specified as command line options for a specific toolkit. In case of the cache simulator, for example, it allows the user to specify parameters for the frontend, the simulator, and the application.

Using this information, DECO builds an execution command for each individual tool and combines this command with the corresponding item in the menu Analysis of the main window. This means, tools can be started with menu 


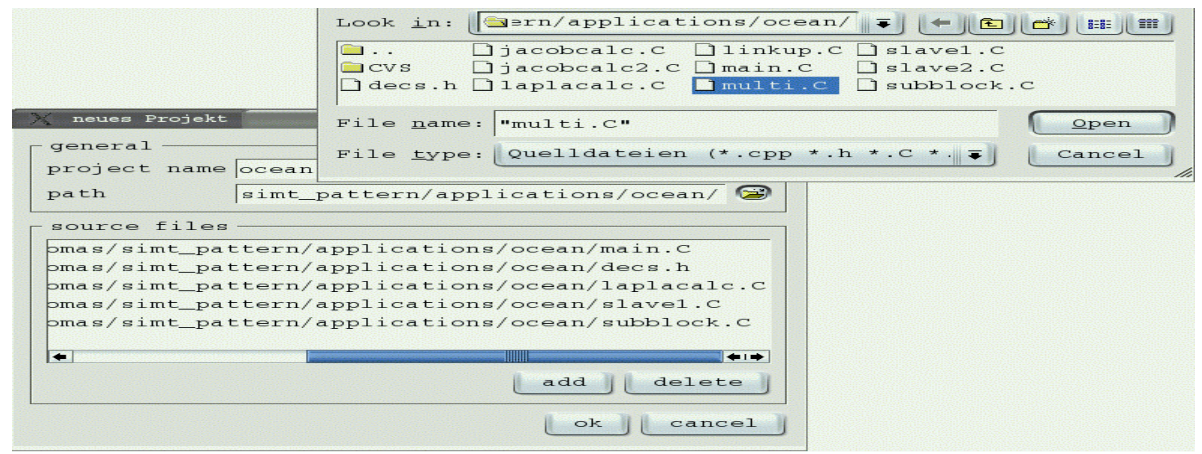

Fig. 2. Creating a new project

choice. During one tool is running, users can configure another tool or edit the applications.

Execution Results. To enable a comparative study of different program versions, DECO visualizes the output of applications. Depending on the application, this output can be execution time, overhead for parallel programs, statistics on memory accesses, or time for synchronization of shared memory applications. The concrete example in Figure 1 visualizes the statistics on cache hits and misses.

\section{Visualizing the Access Pattern}

As mentioned, a specific feature of DECO for our cache locality optimization is the ability of depicting access patterns in the source code. This leads the user directly to the optimization object and more importantly shows the user how to optimize. In the following, we first give a brief introduction of the pattern analysis tool, and then describe the visualization in detail.

Pattern Analyzer. The base of the analyzer [11] is a memory reference trace which records all runtime memory accesses. By applying algorithms, often used in Bioinformatics for pattern recognition, it finds the affinity and regularity between the references, like access chain and access stride. The former is a group of accesses that repeatedly occur together but target on different memory locations. This information can be used to perform data regrouping, a strategy for cache optimization, which packs successively requested data into the same cache block by continually defining the corresponding variables in the source code. The latter is the stride between accesses to neighboring elements of an array. This information can be used to guide prefetching, another cache optimization strategy, because it tells which data is next needed.

Additionally, the pattern analyzer delivers the information whether an access is a cache hit or a cache miss. For a cache miss, it further calculates the push back distance which shows the number of steps a miss access must be put back 
in order to achieve a cache hit. It is clear that users can use this information to change the order of memory accesses for a better cache hit ratio. However, it is difficult to apply this information in its original form based on virtual addresses and numbers. Therefore, DECO provides this specific property of mapping the pattern to the program.

Visualization. Using DECO, patterns are displayed within the source code but also in a separate window for a deeper insight. Figure 3 is an example of the access chain. The window on the right side lists all detected chains with detailed description, including the ID, number of elements, frequency of occurrence, and the initial address. It is also possible to observe more detailed information of a single chain, for example, all accesses contained and the miss/hit feature of each access. This detailed information helps the user decide whether the grouping optimization is necessary.

On the left side access chains are demonstrated in the source code. By clicking an individual chain in the right window, the corresponding variables and the location of references are immediately marked in the program. Users can utilize

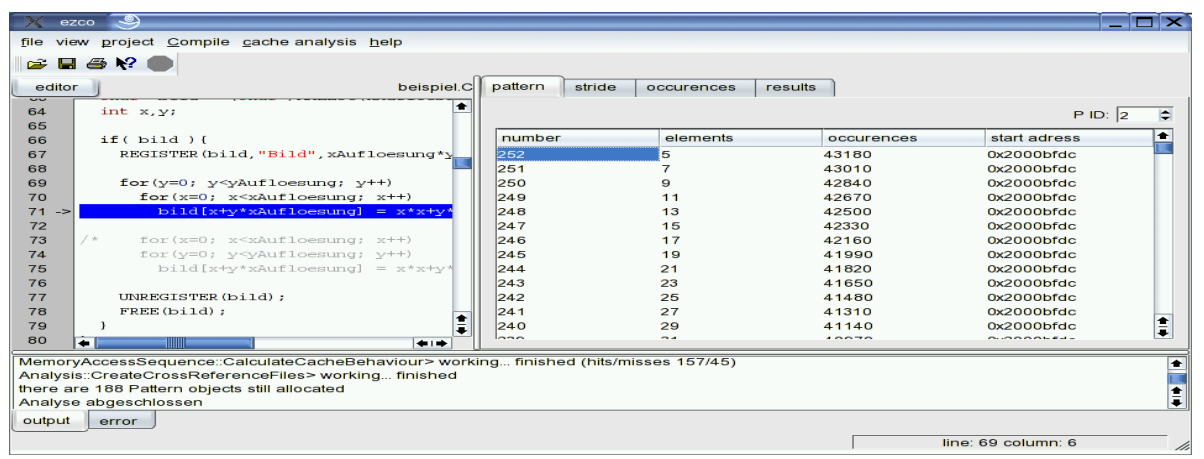

Fig. 3. Presentation of access chains in two windows

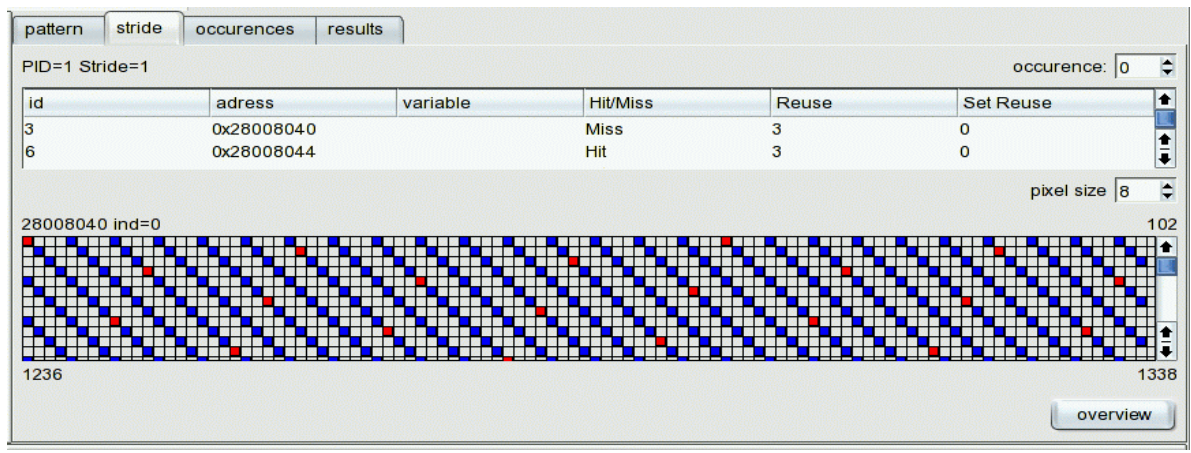

Fig. 4. Visualization of access stride 
this information to move the declarations of the associated variables into a single code line.

Similarly, the push back distance is also combined with the program. For a selected variable DECO marks the first access to it in the code. Then the rest accesses are one-by-one marked with different colors presenting their hit/miss feature. In case of a cache miss, the position, where the reference has to be issued for avoiding the miss, is also marked with color. Based on this information users can decide if it is possible to shift an access. This optimization improves the temporal locality of single variables and is especially effective for variables in loops.

The access stride for arrays can be observed in three different forms. First, an initial overview lists all detected strides with the information about start address, stride length, and the number of occurrence. A further view displays individual strides in detail, with descriptions of all references holding this stride. The third form is a diagram. The lower side of Figure 4 is an example. Within this diagram, all array elements holding the stride are depicted with small blocks. Each small block represents a byte; hence, an element usually consists of several blocks, depending on the type of the array. For example, the concrete diagram in Figure 4 actually demonstrates an array of 4-bytes elements and a stride of length one. The first block of each element is colored in order to exhibit the hit/miss feature. This helps the user reorganize the array structure or change the sequence of accesses to it, if large number of misses had been introduced by this array. The size of blocks can be adjusted using the spin-box at the right corner above the diagram. This allows the user to either observe the global access characteristics of the complete array or focus on a specific region of elements.

Sample Optimization. We show two examples to demonstrate how to use this special feature of DECO for cache optimization. The first code implements a simple scheduler for user-level threads. With this scheduler the threads release the CPU resources by themselves, rather than being evicted after the timeslice. For saving registers by thread swap, a stack is managed for each thread. The current position at the stack has to be recorded in order to restore the registers when the thread acquires the timeslice again. We use a struct to store this stack pointer together with its ID and the stack. By thread switching the stack pointer has to be updated, where the pointer of the old thread has to be written back and the pointer of the new thread is fetched. The declaration of struct and the code for stack pointer update is depicted in Figure 5 .

We perform 1000 switchings between four threads with only the operation of processor release. The trace analysis detects five access chains that repeat more than 1000 times. Observing the chains in more detail, we find that among all accesses to the associated addresses in four of the five only three hits exist with each. Switching to the marked code lines in the source, we further detect that the corresponding accesses are performed for stack pointer update. This leads us to move the definition of stackPointer out of struct and define all the four pointers together. In this case, the number of L1 misses reduces from 4011 to 9. This significant improvement is the result of grouping. 


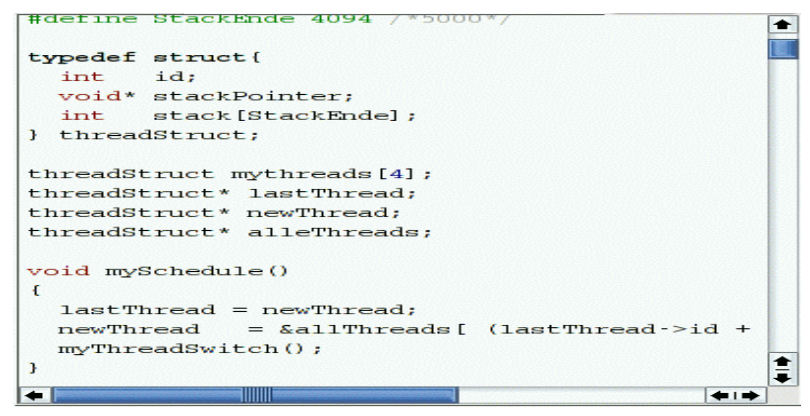

Fig. 5. Source code of the thread scheduler

The second example performs matrix addition. The working set contains three matrices which is declared one after another. Initial run of this code reported 12288 L1 miss without any hits. The trace analysis detected three strides of length one, each corresponding to a single matrix. In principle, for such a stride many accesses shall be cache hit because with a size of 64 bytes one cache line can hold several elements. The only explanation lies in that there exists mapping conflict between all three matrices and this conflict results in the eviction of a matrix block out of cache before the other elements can be used.

To eliminate this conflict, an efficient way is to insert a butter between two matrices so that the mapping behavior of the second matrix is changed. As the applied L1 is a 2-way cache, meaning that a cache line can hold two data blocks, we need only to alter the mapping behavior of one matrix. Here, we put a buffer of one cache line between the second and the third matrix. In this case, only 769 misses has been observed.

\section{Conclusions}

This paper introduces a program development environment that helps the user perform code optimization. This environment is implemented because we note that code optimization requires support of several tools and it is inconvenient to use them separately. Hence, our environment integrates different tools into a single realm. In addition, it provides a flexible interface for users to work with their programs and to study the impact of optimizations. A more specific feature of this environment is that it directly show access patterns in the source code. This allows the user to locate the optimization object and the strategy.

\section{References}

1. D. I. Brown, S. T. Hackstadt, A. D. Malony, and B. Mohr. Program Analysis Environments for Parallel Language Systems: The TAU Environment. In Proc. of the Workshop on Environments and Tools For Parallel Scientific Computing, pages 162-171, May 1994. 
2. H. Brunst, H.-Ch. Hoppe, W. E. Nagel, and M. Winkler. Performance Optimization for Large Scale Computing: The Scalable VAMPIR Approach. In Computational Science - ICCS 2001, International Conference, volume 2074 of LNCS, pages 751760, 2001.

3. J. Dongarra, K. London, S. Moore, P. Mucci, and D. Terpstra. Using PAPI For Hardware Performance Monitoring On Linux Systems. In Linux Clusters: The HPC Revolution, June 2001.

4. J. Fenlason and R. Stallman. GNU gprof: The GNU Profiler. available at http://www.gnu.org/software/binutils/manual/gprof-2.9.1/html_mono/gprof. html.

5. Intel Corporation. Intel Software Development Products. available at http://www.intel. com/cd/software/products/asmo-na/eng/index.htm.

6. P. S. Magnusson and B. Werner. Efficient Memory Simulation in SimICS. In Proceedings of the 8th Annual Simulation Symposium, Phoenix, Arizona, USA, April 1995.

7. B. P. Miller, M. D. Callaghan, J. M. Cargille, J. K. Hollingsworth, R. B. Irvin, K. L. Karavanic, K. Kunchithapadam, and T. Newhall. The Paradyn parallel performance measurement tool. IEEE Computer, 28(11):37-46, November 1995.

8. R. Mueller-Pfefferkorn, W. E. Nagel, and B. Trenkler. Optimizing Cache Access: A Tool for Source-To-Source Transformations and Real-Life Compiler Tests. In Euro-Par 2004, Parallel Processing, volume 3149 of LNCS, pages 72-81, 2004.

9. B. Quaing, J. Tao, and W. Karl. YACO: A User Conducted Visualization Tool for Supporting Cache Optimization. In High Performance Computing and Communcations: First International Conference, HPCC 2005. Proceedings, volume 3726 of Lecture Notes in Computer Science, pages 694-703, Sorrento, Italy, September 2005 .

10. J. Tao and W. Karl. CacheIn: A Toolset for Comprehensive Cache Inspection. In Proceedings of ICCS 2005, volume 3515 of Lecture Notes in Computer Science, pages 182-190, May 2005.

11. J. Tao, S. Schloissnig, and W. Karl. Analysis of the Spatial and Temporal Locality in Data Accesses. In Proceedings of ICCS 2006, number 3992 in Lecture Notes in Computer Science, pages 502-509, May 2006. 